\title{
SoundCloud: sus posibilidades y limitaciones como una plataforma de distribución de pódcast educativos
}

\section{SoundCloud: Possibilities and Limitations of a Streaming Platform for Educational Podcasts}

\author{
Katia Grau Ibarra \\ Universidad Estatal a Distancia. San José, Costa Rica \\ kgrau@uned.ac.cr
}

\begin{abstract}
Resumen
Este artículo plantea una sistematización de experiencias; evalúa y compara algunas ventajas y limitaciones al distribuir pódcast educativos de la Universidad Estatal a Distancia (UNED) a través de SoundCloud, plataforma en línea bajo demanda. Esta fue una búsqueda para seleccionar una plataforma de distribución en línea para ofrecer a los usuarios la consulta y la posibilidad de descarga de los materiales en audio producidos por el Programa de Producción de Material Audiovisual (PPMA) de la UNED. En este contexto, se estudió SoundCloud, sus orígenes, el posicionamiento que ha alcanzado y se evaluaron las herramientas que ofrece. Se hizo primero una revisión bibliográfica y una breve comparación con otras plataformas. Con base en la experiencia al haber utilizado SoundCloud en el Programa de Producción de Material Audiovisual (PPMA) de la UNED, se rescatan algunas posibilidades y limitaciones para distribuir pódcast educativos. A pesar de ser una plataforma comercial, SoundCloud permite la accesibilidad deseada para la difusión de los audios, es asincrónica y atemporal, posibilita el uso de varios dispositivos, genera un espacio de intercambio de información y fomenta algunos aspectos sociales claves para el éxito del pódcasting.

Palabras clave: SoundCloud, podcast, audios educativos, plataformas en línea, uned, distribución recursos educativos
\end{abstract}

\begin{abstract}
This article explains the systematization of experiences that evaluate and compare some advantages and limitations of streaming educational podcasts of the National Distance Education University (UNED, acronym in Spanish) through SoundCloud, an online on-demand streaming platform. This was a search to select an online streaming platform to offer users the possibility to consult and download the audio materials produced by the Audiovisual Material Production Program (PPMA, acronym in Spanish) of the UNED. In this context, we studied SoundCloud, its origins, the position it has gained, and we assessed the tools it offers. First, a bibliographic review was done, followed by a brief comparison with other platforms. Based on the experience of using SoundCloud in the Audiovisual Material Production Program (PPMA, for its acronym in Spanish) of the UNED, we highlight some possibilities and limitations to the distribution of educational podcasts. In spite of it being a commercial platform, SoundCloud offers the accessibility needed for the diffusion of audios, it is asynchronous and timeless, it can be used on different devices, it generates a space for information exchange, and it promotes some social aspects that are key for the success of podcasting.
\end{abstract}

Keywords: SoundCloud, podcasts, educational radio programs, streaming services online, distribution of educational resources, uned 


\section{Introducción}

En la actualidad proliferan las plataformas de transmisión en línea, ya sean de consumo bajo demanda, de transmisión en vivo o de distribución de contenidos audiovisuales como Netflix, YouTube, Spotify, ACAST, iTunes, Cuevana, Popcorn Time, iVoxx, entre otros, los cuales han tomado auge por las muchas ventajas que ofrecen. Algunas presentan características como redes sociales; es decir, como comunidades que permiten desarrollar las capacidades Web 2.0; otras son meras plataformas de contenido por catálogo. Estas herramientas ofrecen facilidades a los usuarios que las han convertido en las preferidas; así pues, atraen a millones de usuarios y, a su vez, han modificado el consumo de los audios y videos de forma asincrónica, atemporal, a fin de generar sus propias listas, conectar con comunidades con los mismos gustos y facilitar la utilización de diferentes dispositivos para ver o escuchar materiales audiovisuales.

El rápido crecimiento de las plataformas de distribución y bajo demanda ha modificado la producción y entrega de materiales multimedia en los medios de comunicación masiva. Al mismo tiempo, ha transformado los hábitos de consumo de los productos audiovisuales. Estos son aspectos que debemos considerar con el objetivo de obtener provecho de la elaboración de pódcast educativos y de ese modo satisfacer las demandas y exigencias que el mercado impone, que no podemos ignorar, como la accesibilidad, la portabilidad, descargas rápidas, multidispositivos y características propias de la Web 2.0, como la conexión con otros pares o especialistas, el trabajo colaborativo, con la posibilidad de ser prosumidor; o sea, productor y consumidor a la vez, "consumidor que se implica de forma activa en el desarrollo de productos" (Sheehan, 2012, p. 23, citado en Quintana, Parra y Riaño, 2017, p. 87).

Estas son algunas de las consideraciones que justificaron la escogencia de trabajar con SoundCloud en el Programa de Producción de Material Audiovisual (PPMA) de la UNED. La pregunta de investigación que orientó la búsqueda de información fue: ¿Las plataformas de audio bajo demanda, como SoundCloud, pueden contribuir a la distribución de los pódcast educativos de la UNED para que los usuarios puedan consultar y descargar los materiales didácticos en audio? Se quiso estudiar sus posibilidades y limitaciones.

Este artículo es un inicio en esta línea de investigación que se está gestando en el PPMA. Tal vez quedarán muchas interrogantes, pero este es un tema que comienza a investigarse el cual, sumado a la experiencia acumulada del PPMA en la producción radiofónica, permitirá rescatar algunas reflexiones y hacer sugerencias. Al final, se proponen nuevas líneas para continuar profundizando más en el pódcasting en la UNED.

Gracias a una pasantía de la UNED, dos colaboradores del PPMA pudieron visitar a RTVE (Radio y Televisión Española) en España con fondos del AMI (Acuerdo de Mejoramiento Institucional de la UNED). En esta visita, realizada en octubre de 2017, se pudo constatar dos situaciones principales: por un lado, el avanzado sistema de redes, donde se trasiega toda la información digital entre estudios, productores y transmisión. Por otro, llamó la atención la apuesta por transmitir en línea y la gran inversión en sus sitios webs. La Radio Española, en RTVE, se divide ahora en cinco emisoras y una de ellas es exclusiva para el formato en línea (Radio 5). Las otras emisoras, luego de su transmisión al aire, disponen de un sistema de pódcast a la carta en el sitio RTVE ${ }^{1}$, ordenados por programas. Su sitio

\footnotetext{
${ }^{1} \mathrm{http}: / /$ www.rtve.es/alacarta/
}

SoundCloud: sus posibilidades y limitaciones como una plataforma de distribución de pódcast educativos. Katia Grau Ibarra. 
web es bastante robusto y visitado, además de que permite un contacto con los seguidores e intercambio de comentarios entre los productores y su público.

A raíz de esta visita, y entre otras razones que se discutirán más adelante, en el PPMA quedó en evidencia lo urgente de dedicarse a mejorar el servicio ofrecido de entrega y distribución de los pódcast de la UNED.

\section{Marco teórico}

Plataformas digitales

Una plataforma digital es un sitio creado para gestionar contenidos. El blog de Redator Rock Content (2019) explica que las plataformas digitales son soluciones en línea para la ejecución de diversas tareas en un mismo lugar. Son espacios en Internet que permiten la ejecución de diversas aplicaciones o programas y así poder satisfacer distintas necesidades. Vizcaíno (2018) argumenta que facilitan el acceso a una amplia gama de bienes y servicios, incentivan la competencia y modifican patrones y hábitos de consumo en distintos mercados. Feijóo (2013) plantea que "las plataformas son una versión sui generis de los mercados multilaterales donde se intenta atraer a todos los interesados: productores de contenido e información, desarrolladores de aplicaciones, fabricantes de soportes, anunciantes y, por supuesto, consumidores" (p. 7).

Pueden generarse plataformas educativas (Moodle, Blackboard), sociales (como Facebook, Instagram y Twitter) o de contenido audiovisual (YouTube, Netflix, Vimeo y Spotify). Entre las posibilidades que ofrecen, la mayoría puede estar interconectadas brindando servicios de las otras. Fernández (2016, p. 84) señala que cada interfaz de plataforma compite con las interfaces que diseña el usuario para organizar su trabajo y su navegación en las redes; así, Facebook nos permite vincular en un mismo espacio lo público y lo privado, lo laboral y lo lúdico, lo informativo y lo ficcional.

Internet ha permitido la proliferación de plataformas que ofrecen el servicio de transmisión en línea y ha desafiado la tradicional televisión y radio, argumenta Schmitz (2015). Además, según el formato, pueden especializarse en plataformas bajo demanda de audio 0 específicamente de video (audio-on demand o video on demand streaming). Schmitz (2015) aclara en su tesis que la transmisión en línea (online streaming) se puede dividir en dos categorías: la transmisión en vivo y la transmisión bajo demanda; la diferencia reside, básicamente, en el tiempo de entrega. Evidentemente, la transmisión en vivo es en tiempo real, como las transmisiones de los noticieros, mientras que la transmisión bajo demanda permite ver o escuchar algún material de forma asincrónica, según su conveniencia. Por otro lado, una plataforma streaming le permite al usuario escuchar o reproducir archivos de audio o de música sin que estén almacenados en su disco duro. Estos archivos están guardados en otros servidores y desde su computadora, con conexión a Internet, el usuario accede a este contenido.

Varios factores han contribuido a esta revolución digital. Por un lado, están los aspectos técnicos y tecnológicos: la cobertura y amplio servicio de telefonía celular, el ancho de banda para la transmisión de datos celulares y de Internet, la adquisición de dispositivos como celulares, tabletas y computadoras. Según la Superintendencia de Telecomunicaciones (SUTEL), "en el año 2017, las suscripciones móviles alcanzaron un total de 8840 342, cifra que representa un crecimiento del 6\% con respecto al 2016" (SUTEL, 2018, p. 74) En Costa Rica se contabilizan casi dos celulares por persona, 1,79

SoundCloud: sus posibilidades y limitaciones como una plataforma de distribución de pódcast educativos. Katia Grau Ibarra.

Página 3 de 16 
celular por persona, según La Nación, en su reportaje especial Radiografía de la calidad de vida en San José. Aquí mismo, con fuentes tomadas de la SUTEL, Cambronero (2018) también establece que el $97 \%$ de la población cuenta con celular o tableta con acceso a internet con una velocidad de 256 bits/s o más.

Por otra parte, las facilidades que brinda la entrega bajo demanda contribuyen a distinguir algunos criterios de selección de consumo por parte de los usuarios; por ejemplo: la posibilidad de consultar los productos culturales en cualquier momento, en cualquier lugar, a cualquier hora y cuantas veces desee repetirlo.

Si bien es cierto son herramientas creadas para el entretenimiento, la entrega de productos culturales u otros fines también ha sido aprovechada para el ámbito educativo. Demski (2012) discute cómo la tecnología puede aportar a la educación personalizada, la cual es toda una metodología de enseñanza y estrategia de aprendizaje. Ella advierte que ingenieros en tecnologías educativas analizaban el Netflix factor para obtener ideas y para personalizar el plan de estudios, según los intereses y habilidades de cada estudiante. En Netflix, esta información queda registrada al navegar y seleccionar una película o serie; luego, la misma plataforma hace recomendaciones al usuario con base en su "gusto". Karen Cator, directora de Office of Educational Technology de los Estados Unidos, es entrevistada por Demski y comenta: "we can learn a lot from these algorithms and from the different methodologies behind using data to provide more directed, personalized, and customized learning experiences for children" (p. 35).

O'Baoill (2009), en su tesis, ya planteaba un cambio en las expectativas de los oyentes en relación con las plataformas en línea, específicamente el pódcasting, que propone también un reto para los productores. Este autor plantea la idea de que estamos en un mundo "bajo demanda" y esto supone valorar las regulaciones, ventajas y limitaciones que conlleva distribuir materiales, ya sea por radio convencional o por Internet. Para el pódcasting, menciona tener presente estos retos y herramientas: sindicalización o suscripción RSS, Mp3 portátiles, la producción de archivos digitales de audio, participación social, producción colaborativa, movilidad y una escucha personalizada (p. 15). La suscripción RSS significa que el usuario es notificado cuando se incluye un archivo nuevo (O'Baoill, 2001, p. 48).

Incluso, en su tesis plantea un debate de si estas plataformas de transmisión en línea iban a suplantar las radios tradicionales; es un debate que hoy todavía se mantiene. Sin embargo, desde el 2001 ya se discuten características y expectativas de los oyentes: realimentación en tiempo real, nuevas formas de interactuar con las audiencias e integrar las contribuciones y este feedback de las audiencias en lo que se produce (O'Baoill, 2009, p. 295). En Internet conviven distintas plataformas que ofrecen contenidos sonoros que satisfacen las necesidades de entretenimiento e información de las audiencias por las que las radios compiten desde sus inicios.

En este sentido, nuestro argumento sostiene que, si las audiencias están acostumbradas a recibir un producto audiovisual de una manera, de la misma forma van a exigir esta misma atención, con estas características, al servicio que ofrece una universidad en la entrega de sus pódcast educativos. Además, hoy, en un mundo donde todo está integrado, será difícil separar lo educativo de lo social, y es justo lo que se puede aprovechar de las plataformas: esta interconexión de servicios para hacer atractiva la entrega de recursos didácticos radiofónicos.

SoundCloud: sus posibilidades y limitaciones como una plataforma de distribución de pódcast educativos. Katia Grau Ibarra.

Página 4 de 16 
RED. Revista de Educación a Distancia. Núm. 59, Artíc. 09, 30-10-2019

DOI: http://dx.doi.org/10.6018/red/60/09

Pódcast

Antes de conocer la plataforma SoundCloud, es fundamental considerar qué es un pódcast. La palabra en sí es la síntesis de dos palabras en inglés: broadcast (transmisión) y iPod, el dispositivo de Apple para escuchar música. Este término fue acuñado por el periodista Ben Hammersley en febrero de 2004, a pesar de que la tecnología existe desde el año 2001. Copley (2007) lo define como un archivo que es distribuido por Internet para ser escuchado en las computadoras personales o en dispositivos móviles.

Cuando se consulta el material bibliográfico en relación con el pódcast y su impacto en los procesos de enseñanza-aprendizaje, es interesante observar lo novedoso que puede resultar este tema, puesto que para el 2010 varios autores admitían, entonces, que no había suficiente evidencia por lo reciente que resultaba este fenómeno (Solano y Sánchez, 2010, p. 126). No obstante, en artículos más recientes, sí se encontró muchas investigaciones muy puntuales en diversos centros de enseñanza a lo largo del mundo para apoyar diferentes materias y en cualquier nivel educativo, sea secundaria o educación superior, como por ejemplo para enseñar idiomas, medicina, turismo y más.

McClung y Johnson (2010) consideraron que los pódcast eran relativamente jóvenes en aquel momento. Para Pot (2013) el pódcasting como un medio es propio de Internet. Según este autor, su historia combina tres tecnologías: audio comprimido, suscripciones automáticas RSS y los dispositivos portables de música (iPod).

Entre las características que definen a un pódcast se encuentra su atemporalidad. Este audio fue creado para ser descargado y escuchado cuando el usuario lo considere pertinente y no para ser transmitido a una hora específica. Comenzó con un sistema de suscripción y seriados; hoy algunos se mantienen así; otros, aunque no estén inscritos en un RSS, también son considerados pódcast, y, además, no es necesario disponer de un iPod para recibir un pódcast (Solano y Sánchez, 2010). Estas autoras señalan que entre las motivaciones originales para crear un pódcast estuvo la intención de que pudiera ser "distribuido como un correo electrónico, y que la descarga de contenidos se pudiera hacer desde dispositivos móviles, personales, disponibles en cualquier lugar y a cualquier momento" (p. 126).

Los pódcast buscan nichos, a sus oyentes, a una comunidad que comparta un tema. Por eso se recomienda que el formato de estos audios sea más íntimo y personal con su audiencia. En la educación a distancia permiten establecer una relación emocional con el profesor, comentan Laaser, Jaskilioff y Rodríguez (2010). Aunque estos autores recomiendan un mínimo de tiempo de 10 minutos de duración, existe controversia y diferentes posiciones al respecto. Según la experiencia de la autora de este artículo, como productora de pódcast educativos, existen otros criterios para definir la duración, como público meta, objetivos y usos pedagógicos. Se coincide en que no debe ser muy largo. No obstante, para un pódcast que se transmite en un soporte digital comprimido (Mp3), en plataformas de transmisión en línea, no hay límite de tiempo porque no hay que cumplir con tiempos establecidos, como en una emisora que lo exige así para cumplir con su parrilla de programación (15 min, 30 min, 1 hora, etc.) en horarios determinados y según el perfil de la emisora.

Y ¿qué se entiende por pódcasting? McClung y Johnson (2010, p. 83) lo definen como el proceso de descargar los pódcast, así como también el desarrollo de programas pódcast.

SoundCloud: sus posibilidades y limitaciones como una plataforma de distribución de pódcast educativos. Katia Grau Ibarra.

Página 5 de 16 
A menudo surge la inquietud acerca del apoyo que pueden brindar los pódcast en la educación a distancia. Laaser, Jaskilioff y Rodríguez (2010) cuestionan si el pódcasting es un nuevo medio para la educación a distancia y concluyen que es útil para cualquier sistema de enseñanza-aprendizaje, ya sea presencial, blended learning o a distancia. No se puede restringir a una metodología específica, pero sí tiene que haber claridad en la variedad de posibilidades pedagógicas y de producción radiofónicas, que se puede distinguir con base en aspectos técnicos, entre quién es el que produce, si es aficionado o profesional, el propósito del pódcast educativo y los objetivos por cumplir con este recurso.

Todavía el pódcasting está evolucionando. Poco a poco el pódcast ha ido tomando más auge. La firma de investigaciones Edison Research, de PEW Research Center, reportó que en Estados Unidos ha habido un crecimiento gradual y lento en la cantidad de personas que escuchan pódcast (Mitchell y Holcomb, 2016). Sin embargo, gracias a la fuerza que alcanzaron plataformas de descarga o de consumo bajo demanda como Netflix, Spotify o YouTube, atrajeron nuevos consumidores y fortalecieron el ver videos o escuchar audios a la carta. Schmitz (2015) señala que "With the digitization of content and the emergence of technological innovations, the consumer behavior is fundamentally changing and eroding traditional revenue flows" (PwC 2014, citado por Schmitz 2015, p.11).

El mismo surgimiento del pódcast ha modificado la forma en que las personas se relacionan con los medios (McClung y Johnson, 2010, p. 82). Los dispositivos portátiles (Mp3, iPods, celulares inteligentes, etc.) y las opciones de sitios y plataformas en línea para escuchar sus audios preferidos, sean pódcast o solo música, han generado, asimismo, nuevos escenarios y nuevas formas de consumir los productos audiovisuales. Las posibilidades de crear sus propias listas o de concentrarse en temas de su interés constituyen hasta una forma muy personalizada de escucha porque además se hace en el momento y lugar de su preferencia.

\section{Antecedentes}

Como antecedente, en el PPMA, desde el 2013, se ofrecía un servicio de pódcast para que los estudiantes descargaran los audios necesarios para sus asignaturas. La dirección del sitio era: www.audiovisuales.uned.ac.cr/mediateca. No obstante, esta plataforma se volvió obsoleta tecnológicamente. Dejó de reproducir los audios y videos, pedía constantemente una actualización del Flash Player y no permitía la consulta de sus materiales desde dispositivos móviles; únicamente se ofrecía sus servicios desde computadoras de escritorio (hoy ya no está activo, está en proceso de rediseño y nueva programación, por el momento el sitio es: https://www.uned.ac.cr/dpmd/audiovisuales/).

\section{Metodología}

Así, para responder a ¿cuál plataforma de distribución en línea cumplía con las necesidades de distribución del PPMA? y ¿las plataformas de audio bajo demanda, como SoundCloud, pueden contribuir a la consulta y descarga de los pódcast educativos de la UNED para apoyar los procesos de enseñanza aprendizaje?, se pretendió obtener una respuesta con base en una revisión bibliográfica y con apoyo en la experiencia al aplicar la plataforma SoundCloud en el PPMA. Para buscar una respuesta a la pregunta de investigación se hizo un análisis documental de artículos, tesis de posgrado y conferencias. Además, se buscó información con base en la puesta en marcha de la misma plataforma SoundCloud y se hizo una comparación entre algunas plataformas populares: YouTube, Spotify, IVoox y Mixcloud.

SoundCloud: sus posibilidades y limitaciones como una plataforma de distribución de pódcast educativos. Katia Grau Ibarra.

Página 6 de 16 
Para el análisis documental, la muestra corresponde a los resultados obtenidos en la búsqueda en cuatro bases de datos: Ebsco, ProQuest, Google Scholar y Science Direct. Se quiso descartar que ya existiera un estudio acerca de la plataforma aplicada en ambientes educativos y no se encontró ninguna en estas bases de datos consultadas. Se quería corroborar si existía alguna investigación académica en relación con el uso de la plataforma SoundCloud para la educación; por eso, se eligió ProQuest para cerciorarse si ya existía alguna tesis, y se eligió Ebsco y Science Direct para buscar algún artículo relacionado con alguna investigación atinente. Consultar Google Scholar permitió aplicar este mismo criterio, pero se ampliaron los resultados a artículos digitales acerca de la programación de SoundCloud, estados financieros, competencia en el mercado y algunas referencias bibliográficas que ya no tenían un carácter académico. Entonces, se seleccionaron las fuentes en inglés y en español enmarcadas entre el 2007 (año de creación de SoundCloud) hasta la actualidad, el 2018.

El criterio de actualidad es importante en tecnología por su rápido desarrollo, ya que aparecen y desaparecen herramientas con celeridad. Por esta razón, por un lado, se encontraron algunos artículos con 10 años de antigüedad, y aun así se percibieron un poco desactualizados; no obstante, presentaron algunos puntos de vista que pretendían pronosticar algunos comportamientos y que hoy permiten hacer una comparación con la realidad. Por otro lado, se posibilita así analizar tendencias o prestar atención a predicciones que todavía hoy generan controversia, como la desaparición de los medios tradicionales (la radio y la televisión).

Se realizó la búsqueda por palabras claves: SoundCloud, SoundCloud y educación, SoundCloud and education, SoundCloud enseñanza aprendizaje, plataformas bajo demanda, streaming on line educational podcasts y streaming services. Se revisó la literatura y se le dio prioridad a las fuentes más actuales, las que permitieran explicar nuestros conceptos claves y las que combinaran, sobre todo, la relación entre plataformas en línea o bajo demanda, el tema de la educación y, principalmente, que hicieran referencia a SoundCloud.

Se utilizaron artículos, tesis y demás material bibliográfico que solo tuviera relación con SoundCloud. Se tuvo que descartar la mayoría de los resultados obtenidos de los pódcast educativos, puesto que se encontraron cientos de investigaciones muy específicas. El tema de los pódcast educativos es muy amplio, dado que se puede abordar desde su producción, aspectos técnicos, su aplicación y usos educativos y en otras áreas, resultados de investigaciones, motivaciones de los usuarios o solo dedicarse al estudio de los dispositivos, entre muchas más delimitaciones. Por tal amplitud temática, se seleccionaron los materiales que tenían alguna relación directa entre pódcast y SoundCloud, y los que fueran necesarios para explicar los conceptos básicos del pódcasting. Se les dio prioridad a los que se refirieran a las plataformas bajo demanda, a SoundCloud, sus posibilidades de uso, críticas de usuarios y pronósticos de expertos en tecnología.

\section{Resultados}

Comparación de plataformas

Con base en la revisión bibliográfica, para decidir cuál plataforma se ajustaba mejor a las necesidades de distribución del PPMA y cuál ofrecía un mejor servicio de descarga, interacción social y conexión con otras redes sociales, se decidió comparar las siguientes

SoundCloud: sus posibilidades y limitaciones como una plataforma de distribución de pódcast educativos. Katia Grau Ibarra. 
opciones: YouTube, Ivoox, Spotify, MixCloud, SoundCloud o un desarrollo propio. Esta última opción se refiere a la contratación externa de unos desarrolladores web para diseñar y programar una plataforma hecha a la medida para el almacenamiento y distribución del PPMA UNED. Sin embargo, esta experiencia ya se había vivido en los años 2008-2009 para el desarrollo de la primera plataforma. Fue un proyecto exitoso cuando se contrató a la empresa privada EI Domo Comunicación. La plataforma de entonces funcionó acorde por 8 años, aproximadamente, con una parte en un soporte Joomla y otra en phpMotion. No obstante, el proceso de su desarrollo tomó más de un año y los costos fueron justos en su momento, pero cuantiosos si hoy se compara hacer esta inversión contra el hecho de solo adquirir una licencia por tener acceso a los servicios de una plataforma ya desarrollada como SoundCloud, Ivoox, BandCamp, Hearthis.at, Pandora y otras. Se compara un monto aproximado por $\$ 2550$ en el primer año de desarrollo de la plataforma del PPMA (2009) versus, hoy día, a una tarifa de $\$ 140$ al año por una licencia de una plataforma, aproximadamente. Si bien tiene su diferencia en el monto, no es del todo descabellado invertir en una plataforma propia si se disponen de medios, ya que tiene ventajas diseñar las herramientas a la medida del público meta y poder tener total control de su administración, algoritmos y motores de búsqueda.

El cuadro 1 muestra un comparativo con base en la información que ofrece cada plataforma en artículos comparativos y bases de datos; en el proceso de selección se contó con el apoyo del Ingeniero de sistemas del PPMA, Minor López González. Cabe aclarar que esta comparación no se hizo para determinar cuál es la mejor plataforma de distribución de audios, sino cuál ofrecía las herramientas que el PPMA necesitaba. Las experiencias son muy diferentes entre cada plataforma. 
RED. Revista de Educación a Distancia. Núm. 59, Artíc. 09, 30-10-2019 DOI: http://dx.doi.org/10.6018/red/60/09

Tabla 1. Comparación entre posibles plataformas para usarse en el PPMA

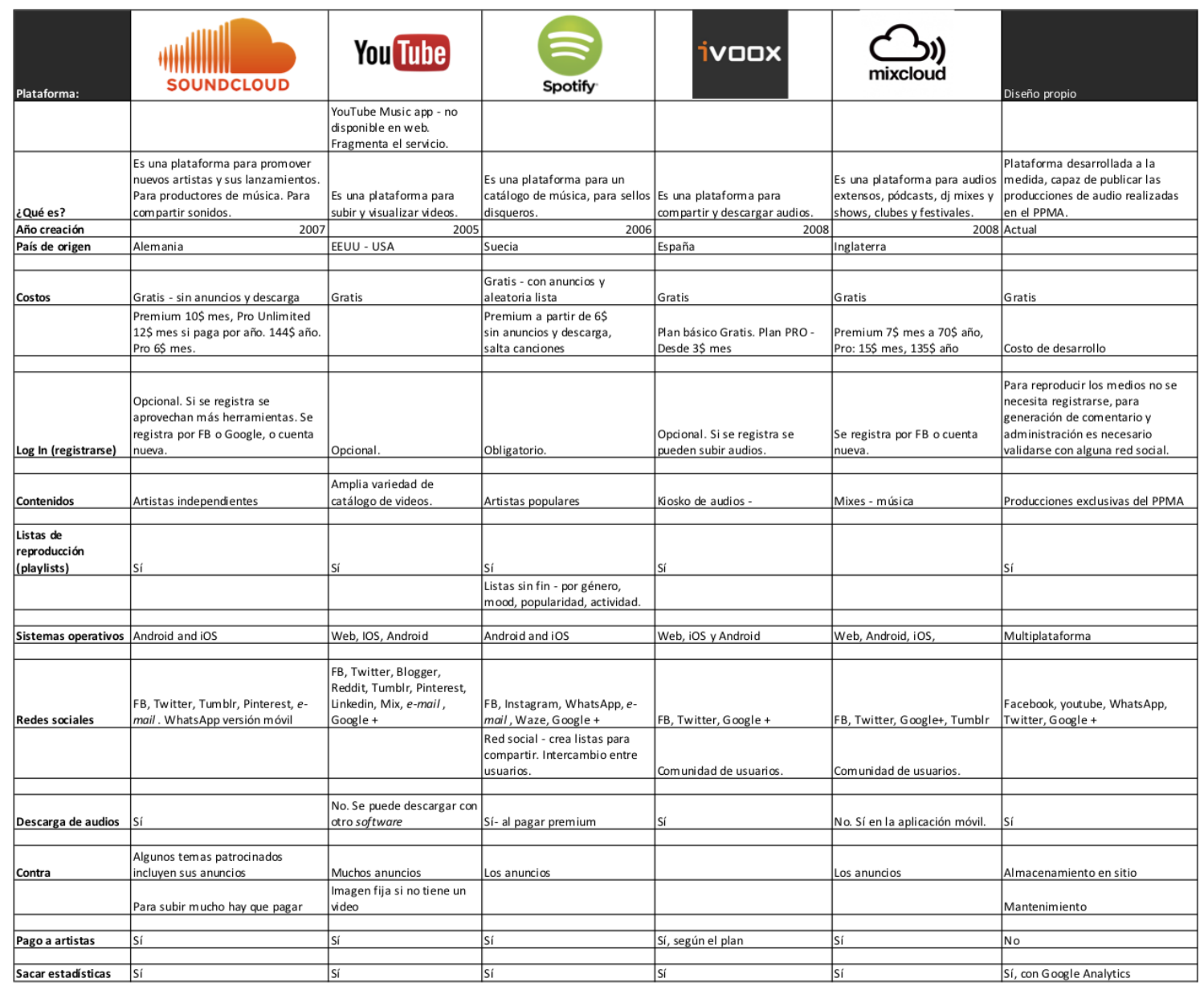

Fuente: Creación propia, a partir de comparaciones generadas en Ondesoft.com, Slant.co, Crunchbase.com, LatinWMG.com, Hossmusic.com

¿Qué es SoundCloud y cuáles son sus ventajas para el PPMA?

El servicio de atención a los estudiantes, tutores, encargados de cátedra, investigadores y demás público usuario obligó al PPMA a dar una pronta solución a la desactualización de la plataforma antigua propia de la oficina y se decidió aprovechar una plataforma de distribución de audio ya creada, como SoundCloud, que cumpliera con las características antes discutidas. Entonces, se creó un perfil con el nombre "Audiovisuales Podcast UNED" para distribuir los audios del PPMA en SoundCloud².

SoundCloud es considerada una de las plataformas más grandes de streaming de audio y opera bajo los principios de gratuidad para sus usuarios, en especial, su audiencia que escucha. A la vez, ofrece planes de pago con algunos beneficios. Fue creada por Alexander Ljung y Eric Wahlforss. (Schmitz, 2015, y Mac, 2013a),

\footnotetext{
${ }^{2}$ www.SoundCloud.com
}

SoundCloud: sus posibilidades y limitaciones como una plataforma de distribución de pódcast educativos. Katia Grau Ibarra. 
Aunque la plataforma fue creada en un principio, en el 2007, para promover la divulgación de música y establecer una red de contacto e intercambio entre productores musicales y consumidores, para el 2013 reportó 250 millones de usuarios inscritos y ha fortalecido algunas herramientas (Dillet, 2013).

El sitio web Techcrunch, en el 2016, reportó que SoundCloud tenía 175 millones de usuarios que escuchaban al mes. SoundCloud y Spotify son las plataformas que más usuarios tienen, Spotify con 75 millones al mes. En algunos artículos y entre asiduos usuarios de plataformas, SoundCloud se considera el "YouTube" en audio (Mac, 2013b). En la columna de la revista Entrepreneur, Handley (2015) comenta la versatilidad que ofrece SoundCloud y las posibilidades multimedia, porque junto a un texto se puede incrustar un audio relacionado con el tema, no textual, sino que amplíe lo escrito (pp. 29-30).

Mac (2013a), en la revista Forbes, califica SoundCloud como una plataforma social de moda que intenta democratizar la música y, además, cualquier experiencia de audio (p. 78). Este autor cita a Lung, el creador de SoundCloud, quien explica: "SoundCloud allows anybody in the world to share audio that they've created, whether it's music, to recordings of their kids on their phone to speeches from Obama". Ljung says. "It also allows anyone to go on the mobile application or the Web and then find these amazing sounds" (p. 78).

Según Schmitz (2015), por el crecimiento de su base de datos, el servicio de SoundCloud se transformó de una plataforma de distribución que comenzó con el lanzamiento de música original y ahora se diversificó a divulgar audios de toda índole, como programas de radio, pódcast o audiolibros, etc. Estaciones de radio tradicionales aprovechan el potencial de la plataforma SoundCloud para difundir sus programas, una vez transmitidos al aire. Los programas son subidos en línea y quedan a disposición del oyente para su descarga o promoción.

La revista Forbes, en el 2017, elaboró una lista de los 41 mejores pódcast intelectuales; así la tituló (Quora, 2017). Se trata de pódcast de ciencia, filosofía, arquitectura, historia, política, impuestos, economía, espiritualidad, lenguaje y muchos temas más.

Las universidades también están aprovechando esta plataforma para colocar sus pódcast: tutorías, conciertos, exposiciones, conferencias y demás material universitario. Como ejemplos se pueden mencionar a la Universidad de Cambridge en Inglaterra, Harvard, Columbia, Yale y Universidad Autónoma de México (UNAM), entre muchas más alrededor del mundo.

En Costa Rica, la Universidad Tecnológica sube su programa radiofónico "ImpactoTec", el cual se transmite los sábados por Radio Monumental 93.5 FM, a las 11:30 a.m. Las radioemisoras de la Universidad de Costa Rica (UCR) tienen su perfil en SoundCloud y suben los programas de sus tres emisoras: Radio Universidad, Radio U y 870 AM.

El Observatorio de Tecnología en Educación a Distancia y el Centro de Investigación en Cultura y Desarrollo (CICDE) de la UNED subieron unos audios en el 2012, el 2015 y el 2017, y crearon su propio perfil, pero desde entonces no parece haber ninguna otra entrada nueva. Onda UNED también subió una única pista en el 2017, pero no subió más audios. Cadenas de noticias también están utilizando SoundCloud, algunas de ellas son: BBC, CNN, RTVE, Repretel CR, Teletica radio, Fox y Caracol.

SoundCloud: sus posibilidades y limitaciones como una plataforma de distribución de pódcast educativos. Katia Grau Ibarra.

Página 10 de 16 
Como se observa, aunque en un inicio la plataforma SoundCloud surgió para atender a los productores de música, hoy el servicio se ha multiplicado y se ha ampliado. Además, no hay anuncios, y si se paga por la licencia se tiene derecho a más servicios y herramientas.

Para resolver la necesidad de distribuir los audios educativos, en el 2018, se implementó trasladar todos los audios a SoundCloud. Se subió todo el material radiofónico a la nueva plataforma, un total de 2000 audios aproximadamente que incluían desde programas de radio hasta producciones académicas producidas por el PPMA. Se ingresó, uno por uno, cada pódcast con su respectiva ficha informativa y una imagen. Esta tarea tomó un tiempo total de tres meses para la ingesta de datos.

Se diseñó, además, una campaña de divulgación para promover la nueva dirección y poder dirigir a los usuarios a la nueva plataforma. En el transcurso de subir los archivos, se empezaron a compartir y utilizar algunos audios ya subidos en SoundCloud. Fue posible obtener algunas primeras impresiones, de forma empírica (vía correo electrónico) de encargados de cátedras, directores de Escuela de la UNED y jefes de otras dependencias, quienes brindaron su apoyo a la decisión de usar una plataforma distinta a la antigua. Ellos comentaron lo fácil que encontraban su navegación. Lo anterior plantea una nueva línea de investigación para un futuro cercano; además, lleva a discutir las ventajas y limitaciones de este tipo de plataformas y su aplicación en contextos educativos, al menos este caso de SoundCloud en el PPMA de la UNED.

Con base en las estadísticas de la misma plataforma SoundCloud, se registraron 64 816 reproducciones de los audios del PPMA desde el 3 de marzo de 2018 al 24 de marzo de 2019. Si hay 2200 pistas subidas, esto se podría traducir en 29 reproducciones al día. Se contabilizaron 143 descargas, donde los meses con mayor movimiento de descargas fueron setiembre y febrero. Por otro lado, los países con más reproducciones fueron: Costa Rica (22 252), Estados Unidos (19 115) y México (7102).

Ventajas de SoundCloud en el PPMA

En el PPMA, se aprovecharon varias de sus funciones que resultaron útiles y valiosas. A continuación, se detallan las razones para justificar por qué se eligió trabajar con SoundCloud a fin de distribuir los materiales de audio de esta oficina y, con base en su aplicación, cuáles ventajas se descubrieron con miras a la entrega de pódcast en la UNED con el propósito de contribuir con la enseñanza-aprendizaje.

- La principal razón: porque es gratis. A los usuarios no se les cobra ninguna tarifa si solo van a escuchar un material, aunque sí se les solicita crear un usuario para registrarse. Esto es necesario para contribuir con la base de datos de los usuarios y para poder utilizar los servicios personalizados de generar sus playlists, sus favoritos, a quiénes están siguiendo y sobretodo poder escucharlo en cualquier dispositivo y darle seguimiento a su cuenta. A los artistas o productores que quisieran ampliar los servicios o herramientas de SoundCloud pueden optar por pagar una tarifa, y así lo está haciendo la UNED: pagó por una licencia para disponer del espacio de derecho a publicación ilimitada para subir todos los materiales del PPMA.

- Otra razón primordial es porque tiene una aplicación para móviles. SoundCloud cuenta con una aplicación (app) que puede ser descargada en cualquier sistema operativo de teléfono inteligente: IOS o Android.

- Por la cantidad de usuarios. En el 2013, SoundCloud reportó tener una suscripción de más de 250 millones de usuarios en casi todo el mundo (Dillet, 2013). Es accesible desde cualquier parte del mundo que disponga una conexión y esta gran cantidad de usuarios

SoundCloud: sus posibilidades y limitaciones como una plataforma de distribución de pódcast educativos. Katia Grau Ibarra.

Página 11 de 16 
ofrece una base sólida de la compañía. Además, al estar más de diez años en línea, se convierte en una plataforma consolidada, sobre todo en un mercado donde aparecen y desaparecen con facilidad este tipo de servicios.

- Es global, así los materiales de la UNED van a tener la distribución y exposición alrededor del mundo.

- Por la red social y su conexión con Facebook, Twitter, WhatsApp y otras redes sociales. Con facilidad se puede compartir un audio en otras redes populares y esto amplía el rango de distribución y promoción de los pódcast de la UNED.

- Por la herramienta de comentarios. SoundCloud permite que el usuario comente sobre un audio determinado. Recibir comentarios facilita obtener realimentación de los usuarios y poder establecer un canal de comunicación. La plataforma ofrece una ventaja que puede ser una herramienta muy atractiva: a lo largo de la onda gráfica del archivo se puede hacer una nota justo en el punto de interés (Gray y Lidsky, 2012, p. 141).

Así, el oyente anota el comentario o pregunta y queda marcado con su nombre de usuario y fecha del día que comentó. Esto permite atender dudas u observaciones de un momento preciso en el audio. También, genera un espacio como si fuera un foro de discusión, lo que podría ser una herramienta útil para el cuerpo docente o para quienes producen. Finalmente, se reciben correos de los usuarios.

- Porque al ser una plataforma de distribución genera bases de datos y estadísticas. Esto permite darle seguimiento al usuario, en alguna medida, y determinar cuáles son los pódcast más escuchados.

- Permite crear listas (playlists). De esta manera, el usuario puede organizar los pódcast de su interés en el orden de escucha deseado y dejar en espera un audio para escuchar luego, sin tener que realizar toda la búsqueda desde el inicio. Además, esta herramienta resultó útil porque muchas producciones en el PPMA son en serie, entonces se decidió colocarlas bajo un título que contiene varios capítulos; otro caso es el de los programas radiofónicos, los cuales se organizaron por año. Así se encuentran las producciones de Hoy con la UNED, Vivir con Valor, Historias Paralelas y otras más, agrupadas por año. A esto se añade la ventaja de poder enlazar un mismo audio en varias listas, sin tener que subirlo la cantidad de veces de las listas donde lo vamos a ubicar. Entonces, un mismo audio se puede compartir para varias asignaturas; no va a ocupar mucho espacio de memoria en el servidor, ya que ha sido subido una única vez; además, se evitan las confusiones al no estar duplicado o no hay que hacer una doble publicación.

- Mientras un audio se reproduce, la plataforma permite continuar realizando búsquedas u otras funciones sin detener la reproducción de lo que se está escuchando.

- En el momento subir los audios a la plataforma SoundCloud, se pueden subir al mismo tiempo hasta cinco o seis archivos, y esto agiliza la subida de material.

- Ofrece la opción de hacer público o privado un archivo de audio. Esto facilita subir un material y enviárselo al tutor o encargado de cátedra para su aprobación, antes de hacerse público y accesible a todos.

- Como cualquier pódcast por suscripción, envía una notificación a sus seguidores cuando un nuevo archivo ha sido subido. Así se mantiene al día informado de las últimas actualizaciones de los audios de la UNED.

- Tiene un espacio asignado para adjuntar una imagen para las pistas o las listas y encontramos que esto contribuye con el posicionamiento. Es significativo poder asociar un audio con una imagen. En los casos en que se dispone de un diseño específico para una producción o una campaña, esta imagen particular se distingue entre las que no cuentan con un diseño, porque en su lugar se coloca el logo de Audiovisuales Podcast UNED.

SoundCloud: sus posibilidades y limitaciones como una plataforma de distribución de pódcast educativos. Katia Grau Ibarra.

Página 12 de 16 
- Cada audio se identifica con su título, su imagen, una sinopsis y las etiquetas. Toda esta información unificada en un espacio proporciona datos útiles del contenido del pódcast. Un aspecto por resaltar es la posibilidad de anotar dos palabras como una etiqueta. Por ejemplo: movimientos sociales, contaminación sónica, o anotar a los invitados o a las personas clave de la producción por nombre y apellido: Silvia Poll, Frankling Chang, Carlos Luis Fallas, etc.

- Esto nos lleva a otro buen argumento para que una universidad trabaje con SoundCloud: el almacenamiento se hace en un servidor en la nube. No es necesario disponer de equipos ni preocuparse por administrar espacio disponible, tanto en personal calificado como en espacio físico. Es una preocupación menos.

- Se puede programar la fecha de publicación de un pódcast. Primero, se sube y, luego, se planifica que se publique con un día y una hora específicos. De esta manera, según alguna actividad académica, estreno al aire, agenda de la asignatura, se puede programar previamente coordinado con el encargado de cátedra o tutores.

Desventajas y limitaciones de SoundCloud aplicado en el PPMA

- Los programas de música o los que contienen temas musicales algunas veces son censurados y SoundCloud no permite su publicación por criterios de respeto a las licencias y derechos de autor; hay que demostrar que se tienen los permisos. La plataforma, incluso, sanciona al usuario después de varias faltas como esta y no permite volver a subir pódcast por un tiempo determinado; es lo mismo que sucede con YouTube.

- El sistema de búsqueda no es tan preciso: realiza las búsquedas en su sistema global y los resultados no son tan rigurosos. Es muy importante trabajar, entonces, los tags o etiquetas, la sinopsis y el título. Se encontró que el motor de búsqueda se guía más por el título y por la sinopsis; funciona al contrario de muchos otros motores de búsqueda, en los que su principal insumo son las etiquetas. En SoundCloud se detectó que las etiquetas no están cumpliendo a cabalidad esta función para el motor de búsqueda y los algoritmos de búsqueda pueden responder a intereses comerciales que administra la misma plataforma, que de alguna manera podría invisibilizar los materiales de uno.

- Aunque permite crear listas y álbumes, no admite la posibilidad de ordenar y presentar el material con otros criterios. Por ejemplo, si en la UNED se quiere ordenar los pódcast en un menú por asignaturas, no se podrían generar nuevos espacios. Habría que atenerse a los menús y las opciones que ya ofrece, no se pueden cambiar. Asimismo, al hacer la ingesta, los materiales van quedando en orden cronológico, por la fecha de ingreso. Por lo tanto, se trabaja muy bien con el enlace directo del podcast, pero si alguien quiere buscar algo por tema puede perderse y no encontrar la producción de Audiovisuales de la UNED.

- Se han mencionado las ventajas de la aplicación para dispositivos móviles, pero hay que tener conexión a Internet para beneficiarse de sus herramientas. Además, para que las descargas se guarden en la memoria del celular hay que descargar otra aplicación, con el fin de no tener que depender de la conexión.

Hay que tener en cuenta esta consideración con la ley de cobro por descarga: ¿cómo afecta a los usuarios y sobre todo en el área educativa? ¿Cómo impacta el acceso a los materiales educativos?

\section{Conclusiones}

SoundCloud puede contribuir en la distribución de los pódcast educativos de la UNED para apoyar los procesos de enseñanza-aprendizaje; encontramos herramientas útiles que contribuyen con la divulgación de los materiales. Lo más importante es que cualquier

SoundCloud: sus posibilidades y limitaciones como una plataforma de distribución de pódcast educativos. Katia Grau Ibarra.

Página 13 de 16 
recurso educativo esté disponible y sea de fácil acceso, y SoundCloud cumple con estas características. El hecho de poder compartirlo a través de redes sociales contribuye con su promoción, ya sea entre los mismos estudiantes o de parte del profesor hacia los estudiantes. La posibilidad de descarga resulta indispensable para que el usuario lo guarde en el dispositivo de su preferencia y no tenga que depender de la conexión a Internet para escucharlo las veces que considere necesario.

Aún faltan más investigaciones para sustentar cómo las plataformas de audio bajo demanda son empleadas en entornos educativos. Aquí quedan abiertas nuevas líneas de investigación; por ejemplo, determinar el uso de esta plataforma por parte de los usuarios, en especial estudiar al público UNED: estudiantes y cuerpo docente y académico. Investigar si lo encuentran tan amigable para navegar; si tienen alguna preferencia en relación con el dispositivo al momento de estudiar; y también analizar la duración de este pódcast y la entrega en series, por capítulos. No obstante, con base en la experiencia y en la puesta en práctica, aunado al haber empleado una plataforma propia en el PPMA desde el 2010 y luego hacer el cambio a una plataforma comercial como SoundCloud en el 2018, fue posible realizar este análisis comparativo de las herramientas y plantear algunas ventajas y desventajas para el campo de la educación. Se considera un acierto recurrir a tecnologías populares, a herramientas con las que el público en general está familiarizado, sobre todo en cuanto a destrezas de la Web 2.0 se refiere, porque contribuye a que la entrega de los pódcast de Audiovisuales UNED sea accesible, portátil y facilite la navegabilidad por el sitio.

Por otra parte, es importante las interconexiones entre plataformas como la de SoundCloud con Facebook, WhatsApp, Google y otras que contribuyen con la divulgación.

El pódcasting es un recurso didáctico con gran potencial. El objetivo principal es que los estudiantes y otros usuarios puedan encontrar, utilizar y compartir el recurso didáctico radiofónico para apoyar sus procesos de enseñanza y aprendizaje. Las plataformas bajo demanda permiten el acercamiento a estos materiales de forma asincrónica, atemporal, disponible para varios dispositivos, para poder estudiar con estos pódcast desde cualquier lugar y en cualquier momento.

Presentación del artículo: 9 de enero de 2019

Fecha de aprobación: 1 de octubre de 2019

Fecha de publicación: 30 de octubre de 2019

Grau Ibarra, K. (2019). SoundCloud: sus posibilidades y limitaciones como una plataforma de distribución de pódcast educativos. RED. Revista de Educación a Distancia, 60. DOI: http://dx.doi.org/10.6018/red/60/09

\section{Financiación}

Esta investigación no ha recibido ninguna subvención específica de los organismos de financiación en los sectores públicos, comerciales o sin fines de lucro. 


\section{Referencias}

Cambronero, N. (2018). Radiografía de la calidad de vida en San José. La Nación. [Reportaje especial]. Recuperado de https://bit.ly/2PZDeaS

Copley, J. (2007). Audio and video podcasts of lectures for campus-based students: production and evaluation of student use. [Pódcasts de audio y video de conferencias para estudiantes del campus: producción y evaluación del uso del alumno.] Innovations in Education and Teaching International, 44(4), 387-399. DOI: 10.1080/14703290701602805

Costa Rica. Superintendencia de Telecomunicaciones. (2018). Estadísticas del sector de telecomunicaciones. Recuperado de: https://bit.ly/2P4BDi7

Crunchbase. (2019). Spotify. https://www.crunchbase.com/organization/spotify\#section-overview

Demski, J. (2012). This Time It's Personal. [Esta vez es personal] THE Journal (Technological Horizons In Education), 32-36. Recuperado de https://bit.ly/2GInp61

Dillet, R. (29 de octubre de 2013). SoundCloud now reaches 250 million listeners in its quest to become the audio platform of the web. [SoundCloud ahora llega a 250 millones de oyentes en su búsqueda para convertirse en la plataforma de audio de la web] Techcrunch.com. Recuperado de https://tcrn.ch/2tmrRiZ

Espada, A. (2018). Nuevos modelos radiofónicos: las redes de pódcasts en Argentina: producción, distribución y comercialización de la radio on demand. Question, 1(59), e081. doi:https://doi.org/10.24215/16696581e081

Feijóo, C. (2013). Soportes digitales y transformación de las industrias de contenido. El profesional de la información, enero-febrero, v.22, n.1, pp. 5-9. Recuperado de: http://oa.upm.es/25810/1/INVE_MEM_2013_160705.pdf

Fernández, J.L. (2016). Plataformas mediáticas y niveles de análisis. Inmediaciones de la comunicación, 11, 71-96.

Hoss. (24 de marzo de 2016). SoundCloud vs. Mixcloud. Recuperado de: https://www.hossmusic.com/soundcloud-vs-mixcloud/

Gray, T. \& Lidsky, D. (2012). SoundCloud: For Giving the Internet a Voice. [SoundCloud: para darle una voz a Internet] Fast Company,163, 140-141.

Handley, A. (2015). New ways to connect. [Nuevas formas de conectarse] Entrepreneur, 43, 28-30.

Laaser, W., Jaskilioff, S. L. \& Rodríguez Becker, L. C. (2010). Pódcasting: ¿Un nuevo medio para la Educación a Distancia? RED - Revista De Educación A Distancia, 23, 1-11. Recuperado de www.um.es/ead/red

Mac, R. (6 de mayo de 2013a). Breaking the Sound Barrier. [Rompiendo la barrera del sonido] Forbes, 191, 78.

Mac, R. (16 de abril de 2013b) SoundCloud Manages Criticism as It Moves To Become The YouTube Of Audio. [SoundCloud administra la crítica a medida que se convierte en el YouTube de audio.] Forbes. Recuperado de https://bit.ly/2lejd0k

Malavé, M. (09 de febrero de 2019). Las 10 plataformas más rentables de música. Recuperado de https://latinwmg.com/las-10-plataformas-de-musica-digital-masrentables/

McClung, S \& Johnson K, (2010) Examining the Motives of Podcasts Users. [Examinando los motivos de los usuarios de pódcasts]. Journal of Radio \& Audio Media, 17:1, 8295. DOI: 10.1080/19376521003719391

SoundCloud: sus posibilidades y limitaciones como una plataforma de distribución de pódcast educativos. Katia Grau Ibarra. 
Mitchell, A. y Holcomb, J. (15 de junio de 2016) Podcasting: fact sheet. [Pódcasting: hoja informative] State of the News Media 2016. Pew Research Center. Recuperado de https://pewrsr.ch/2SACfCZ

O'Baoill, A. (2009) Broadcasting in an On-demand World Creating Community Radio in the Era of Podcasting and Webcasting. [Transmitiendo en un mundo bajo demanda creando radio comunitaria en la era de pódcasting y webcasting] (Tesis doctoral University of Illinois at Urbana-Champaign) De la base de datos Proquest Dissertations and Theses (UMI 3392239)

Ondesoft. (2019). SoundCloud vs. Spotify: a side-by-side comparison. Recuperado de: https://www.ondesoft.com/spotify-converter-for-mac/spotify-vs-soundcloud.html

Pot, J. (10 marzo de 2013): 'The Evolution of The Podcasts - How A Medium Was Born'. [La evolución del pódcast: cómo nació un medio] Make Use Of. Recuperado de https://bit.ly/2GLgicX

Quintana, B., Parra, C. y Riaño, J.P. (2017). El pódcast como herramienta para la innovación en espacios de comunicación universitarios. Anagramas, 15(30) 81-100.

Quora, Etip Sinid (2017). What are the best intellectual podcasts on SoundCloud? (2017, 2 de marzo) [¿Cuáles son los mejores pódcast intelectuales en SoundCloud?] Forbes (online) Recuperado de https://bit.ly/2tpzNjl

Redator Rock Content (14 de febrero de 2019). Plataformas digitales: Qué son y qué tipos existen? [Mensaje de blog]. Recuperado de https://rockcontent.com/es/blog/plataformas-digitales/

Schmitz, S. P. (2015) Developing a Business Model for a Podcasts Streaming Service. [Desarrollo de un modelo comercial para un servicio de transmisión por secuencias de pódcast] Master's Thesis (M.Sc.) in Media Management at the School for Computer Science and Communication, Royal Institute of Technology. Estocolmo, Suecia. Recuperado de https://bit.ly/2Gp8iPH

Solano, I., y Sánchez, M. (2010). Aprendiendo en cualquier lugar: el pódcast educativo. Pixel-Bit. Revista de Medios y Educación, 36, 125-139. Recuperado de https://bit.ly/2GspJP9

Slant. (2019) Spotify vs SoundCloud detailed comparison as of 2019. Recuperado de https://www.slant.co/versus/733/739/ spotify_vs_soundcloud

Vizcaíno, D. (10 de mayo de 2018) Las plataformas digitales han llegado para quedarse. El país. [Negocios] Recuperado de https://bit.ly/2BBNcJX 